\title{
Linx
}

Revue des linguistes de l'université Paris X Nanterre

$7 \mid 1995$

Saussure aujourd'hui

\section{La barre et la topologie lacanienne}

Jeanne Granon-Lafont

\section{(2) OpenEdition}

Journals

Édition électronique

URL : http://journals.openedition.org/linx/1245

DOI : $10.4000 / \operatorname{linx} .1245$

ISSN : 2118-9692

\section{Éditeur}

Presses universitaires de Paris Nanterre

\section{Édition imprimée}

Date de publication : 1 septembre 1995

Pagination : 439-445

ISSN : 0246-8743

\section{Référence électronique}

Jeanne Granon-Lafont, « La barre et la topologie lacanienne », Linx [En ligne], 7| 1995, mis en ligne le 25 juillet 2012, consulté le 10 décembre 2020. URL : http://journals.openedition.org/linx/1245 ; DOI : https://doi.org/10.4000/linx.1245

Ce document a été généré automatiquement le 10 décembre 2020.

Département de Sciences du langage, Université Paris Ouest 


\title{
La barre et la topologie lacanienne
}

\author{
Jeanne Granon-Lafont
}

1 Que la cure ne puisse se passer que dans une langue particulière (ce qu'on appelle: positive), même à jouer de la traduire, y fait garantie " qu'il n'y a pas de métalangage » selon ma formule. L'effet de langue ne s'y produit que du cristal linguistique. Son universalité n'est que la topologie retrouvée, de ce qu'un discours s'y déplace. L'accès topologique y étant même assez prégnant pour que la mythologie s'y réduise à l'extrême »'.

2 La psychanalyse a un savoir sur la langue, ce savoir sur la structure a grandi et s'est nourri de cent ans de cures psychanalytiques, envisagées ici comme terrain d'expérience, ou du moins au sens de Milner, d'observatoire ${ }^{2}$. Il ne s'agit pas comme en linguistique d'une expérimentation par l'exemple mais d'une prise en compte de l'expérience des cures et de leurs effets comme solide de référence. Toute la fonction de la parole peut nous échapper, si nous nous contentons d'en faire une communication, ou l'expression d'une pensée. La langue est opératoire.

« La linguistique livre le matériel de l'analyse, voire l'appareil dont on y opère. Mais un domaine ne se domine que de son opération. L'inconscient peut être comme je le disais la condition de la linguistique. Celle-ci n'en a pas pour autant sur lui la moindre prise $»^{3}$.

Elle se constitue en fabriquant la position du sujet par rapport à la vérité : aspect de la langue que, derrière Charles Williams Morris, (associé à Carnap à Chicago) il faudrait appeler la linguistique pragmatique, positiviste, voire empirique. Il s'agirait de l'étude de la langue dans ses rapports avec l'utilisateur, selon le linguiste. Ce rapport pourtant se retourne pour la psychanalyse en "étude du sujet dans ses rapports avec la langue». Cette dialectique de constitution, par une seule opération de deux choses, est pensée par Lacan comme coupure.

3 «Coupure épistémologique » dit-on, mais ce n'est pas comme épistémologique que la formule la topologie. Lacan parle de topologie pour la première fois dans le séminaire sur l'Identification en 1962. En 1973 seulement dans l'article l'« Etourdit » le rôle central de la bande de Moebius est assuré et argumenté comme coupure, autre nom de la barre, selon mon hypothèse. 


\section{La barre chez Freud}

La barre a une histoire, elle assurément écrite chez Saussure, mais quelle place a-t-elle dans les écrits de Freud ? Elle apparaît dans « Le mot d'esprit et ses rapports avec l'inconscient » pour expliquer un calembour.

On introduisait dans un salon parisien un jeune homme que l'on disait parent du grand J.J. Rousseau et qui, du reste, portait ce nom. De plus il était roux. il se montra si gauche que la maitresse de maison lança à son introducteur cette épigramme: «Vous m'avez fait connaitre un jeune homme roux et sot, mais non pas un Rousseau ». Et il se mit à rire. C'est d'après la nomenclature classique, un calembour et même des plus mauvais, qui joue sur un nom propre... [et Freud de déplorer la pauvreté technique de ce mot, et de conclure]. Tout se réduit et se borne au calembour Rousseau/roux sot. $»^{4}$

Dans les pages précédentes, Freud organise son texte sur deux ou trois lignes, mais sans barre, et les correspondances sont verticales, comme dans l'exemple fameux :

\section{«FAMI LI ERE MI LIONNAIRE FAMI LIONNAIRE» 5}

Shéma note 5

6 Il le répète quatre fois sur des exemples différents. Puis il définit ainsi le procédé général : "L'emploi du même mot à l'état complet, puis à l'état dissocié. ${ }^{6}$.

«Des combinaisons dont le nombre apparait a priori comme incalculable, permettent d'employer dans une phrase le même mot ou le même matériel verbal, en jouant sur la multiplicité de leurs sens. ${ }^{6}$ »

7 Ce « matériel verbal « est un terme que l'on retrouve dans « La science des rêves » et dans les «Etudes sur l'hystérie $»^{7}$. N'est-il pas aussi une expression de Saussure, pour définir le signifiant?

La question de la rencontre manquée devient donc non pas la définition du signifiant mais son rapport avec le signifié : place occupée par le signifiant «arbitraire». C'est important. Par ce concept en effet, passe la distinction entre science et pseudo-science ${ }^{8}$. Freud n'utilise pas ce terme. Mais il met en jeu à cet endroit le jeu du « sens dans le nonsens ", jeu auquel se livrent les enfants au moment de leur entrée dans la langue.

«L'esprit débute à la façon d'un jeu qui cherche son plaisir dans le libre emploi des mots et des pensées. ${ }^{9}$ "

De « libre » à « arbitraire », il y a l'histoire et la question du plaisir certes, mais surtout ce décalage dévoile l'enjeu d'un autre programme de recherche, la constitution du sujet, linguistique pragmatique? 
10 Positivement la différence intéresse la conception de la production du sens. Lacan reprendra la question là : en quoi la production de sens intéresse la définition du signifiant et celle du sujet?

\section{La barre franchie}

Le sens est l'effet d'un franchissement de la barre saussurienne. Ces conditions sont topologiques. Elles renvoient à la consistance de cette barre comme limite, de la nature de la limite en jeu. La barre est la coupure moebienne. Il est nécessaire de prendre la perspective de sa nouveauté dans l'entendement. Le franchissement s'oppose au métalangage. Cette nouveauté qu'offre la bande de Moebius tient à une formalisation sans métalangage. La formalisation procède toujours d'une généralisation, mais la topologie propose une généralisation particulière qui pourrait se définir comme une formalisation par le trou.

Le métalangage est une terme de logique qui s'appuie sur une stratification des niveaux de langue. Cette stratification peut être reconduite a l'infini (cf. les arbres chomskyens) ou être décrite comme moebienne : un temps il y a deux strates, deux faces, signifiant et signifié séparés par un rapport arbitraire ou libre, le temps suivant, si les deux strates se maintiennent comme place, le signifiant et le signifié s'échangent.

«La métaphore se place au point précis où le sens se produit dans le non-sens, c'est à dire à ce passage dont Freud a découvert que, franchi à rebours, il donne lieu à ce mot qui en français est «le mot» par excellence le mot qui n'y a pas d'autre patronage que le signifiant de l'esprit, et où se touche que c'est sa destinée même que l'homme met au défi par la dérision du signifiant ${ }^{10}$.

Mais trêve d'affirmations, essayons d'argumenter.La formule de la métaphore selon Lacan est la suivante :

$\ll$

$$
f\left(\frac{(S)}{(S)}\right) S=S(+) \text { s. } 11 \text { " }
$$

Shéma note ${ }^{11}$

où $S$ et $S$ ' renvoient aux signifiants, $S$ au sens et le signe $(+)$ au franchissement de la barre. Un signifiant $S$ est représenté par une autre signifiant $S$ '. La métaphore consiste à supprimer ce premier signifiant $S$ qui du coup reste présent dans son voilement et apporte un plus de sens au mot qui le remplace. l'effet poétique tient à la suppression d'un mot à sa place. Lacan propose d'y lire l'effet du franchissement de la barre saussurienne dans la mesure où ce signifiant disparu fait office pour l'autre de signifié. La métaphore précise un signifié qui n'est plus un flux continu et vague de la réalité des objets, mais un sens, une substantifique moelle à découvrir. La barre est franchie parce que l'arbitraire qui préside au rapport entre signifiant et signifié est ainsi a la fois utilisé par l'acte du poète qui peut choisir n'importe quel mot et dénié puisqu'il y a production de sens.

Cette organisation est aussi pensée par Lacan, ainsi en 1966 : 


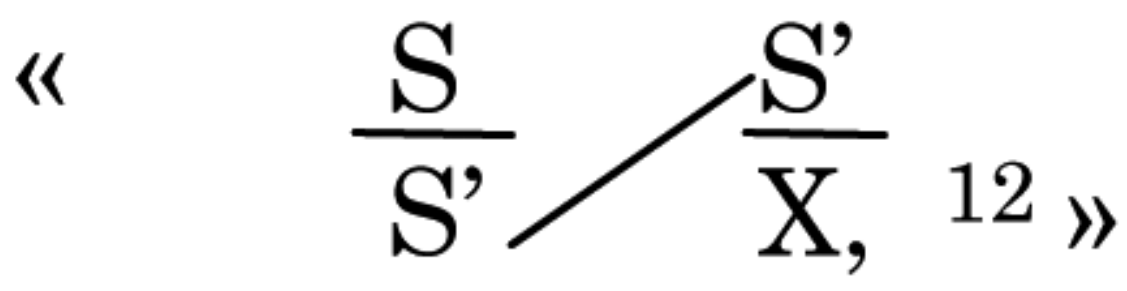

Shéma note ${ }^{12}$

15 où se dessine le franchissement. Il est possible alors d'écrire la formule de la métaphore sur la bande de Moebius, le franchissement de la barre est écrit dans la mise à plat, de la structure, par le dessus-dessous.

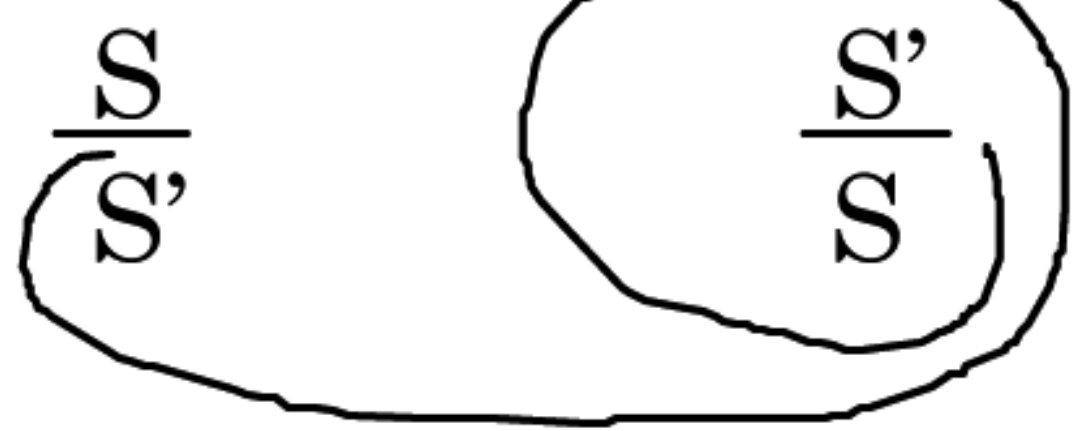

16 Elle peut être écrite partout, et n'a pas de place assignée, sauf peut-être à rebours dans les effets de symptôme d'un sujet.

17 La question devient que toute production de sens est produite par le même processus, c'est une antinomie si on reste au niveau de ce franchissement de barre.

18 Le métalangage se démontre comme un passage à l'infini de la barre saussurienne. Au contraire la coupure moebienne, localement, n'a pas les mêmes propriétés que généralement. Le processus de la métaphore est généralisée comme entourant un trou, celui de la référence. Dans la mise en lettre qu'effectue le métalangage la barre saussurienne fonctionne méconnue dans la mesure où elle est infinitive. Cette logique n'est pas caduque, elle rate l'hypothèse de l'inconscient. Faut-il rappeler les difficultés des arbres de Chomsky dans les auto-croisements de niveaux.

Quand reste-t-on dans le local, avec une barre infranchissable? Quand la généralisation s'impose-t-elle, et produit-elle un effet de sujet, qui met en jeu la structure de la langue en entier, avec son trou, l'inconscient?

« les mots sont dépourvus de sens qui leur soit assignables en propre, le sens propre n'est qu'un préjugé».

21 La barre saussurienne devient ainsi, au delà de la barre du calembour de Freud, pensé comme un artifice de présentation, par ce développement lacanien d'une topologie opératoire, le bord d'une bande de Moebius. Et du coup les mathématiques rentrent dans la linguistique, garante d'une scientificité.

«Sans l'éruption de l'inconscient pas moyen que la linguistique sorte du jour douteux dont l'Université du nom des sciences humaines fait encore éclipse à la science $»^{12}$. 
22

pparaît en fond la question de la limite? Jusqu'à quel point l'écart est-il permis l'arbitraire dénié ? Cette limite dans la langue est absolument conséquente à la constitution du sujet. Ce qui fait éclair pour l'un, fait non-sens pour l'autre : tout le monde ne comprend pas les jeux de mots, et les poètes considérés comme abscons, Mallarmé, ou le travail de Saussure sur les poètes saturniens. Quelle limite à l'arbitraire? L'unilatère de la bande de Moebius répond que cette limite ne peut être pensée seulement dans des catégories spatiales mais doit être pensée dans des catégories temporelles. La différence entre franchissement et séparation tient au nombre de tours, ce qui veut dire le temps de les faire.

La coupure inaugurale et fondatrice de Saussure est certes la barre mais infinitive et il y aurait peut-être intérêt à la penser aujourd'hui comme regroupant deux coupures, la barre entre signifiant et signifié mais aussi la barre entre local et général. Aussi Lacan parlera-t-il de cet «arbitraire » qui spécifie la barre en tant qu'elle dit le rapport entre signifié et signifiant (qu'il n'y a pas, comme « lapsus « pour Lacan ${ }^{13}$. I1 y démontre alors le discours universitaire dans son rapport de soumission au discours du Maître. La bande de Moebius est coupure dans l'espace du temps, barre saussurienne, non pas répétée dans l'étagement sans fin d'une coupure inaugurale et fondatrice mais dans une reconnaissance de son "indépassable» continu, où l'universel ne s'oppose pas au particulier d'un cas, mais au local, opposé à l'ensemble.

\section{NOTES}

1. Jacques Lacan, interview de Jacques Lacan par la radio belge en 1970, paru sous le titre «Radiophonie» in Silicet 2/3, Seuil, 1970, p 64.

2. Jean Claude Milner, «Introduction à une science du langaqe» Seuil, Paris, 1989, p. 50.

3. Jacques Lacan, «Radiophonie» in Silicet 2/3, Seuil, 1970, p 62.

4. Sigmund Freud, Le mot d'esprit et ses rapports avec l'inconscient, Idées Gallimard, 1978 p 47 à 49. La première édition date de 1905, une traduction anglaise parait en 1917 à New York, et la traduction française en 1930.

5. Idem, p 30.

6. Idem, p 51.

7. Les deux livres sont publiés en Français aux PUF. Les premières éditions datent, respectivement de 1900 et 1895 . Pour préciser le rapport, rappelons ici des dates connues : Le Cours de linguistique générale de Ferdinand de Saussure parait en 1916, il reprend une période d'enseignement qui s'étend de 1907 à 1911. Saussure devait parler l'allemand, puisqu'il fait ses études à Leipzig. Le latin est-il encore la langue des universités? Mais il enseigne en français. Il aurait eu la possibilité avant son cours, de lire ce Rousseau/roux sot. Voilà pour les précisions historiques.

8. Présentation du colloque «La linguistique fantastique» organisé par l'E.N.S. de Fontenay en 1983. Publié sous ce titre par Denoël, 1985. «Ce qu'on entend par arbitraire du langage..., n'est rien d'autre que l'axiome par lequel on décrète qu'on ne prendra pas en considération les faits plaidant en faveur d'une ressemblance universelle. Le rejet de l'origine des langues et celui de la motivation du matériel linguistique sont un seul et même acte épistémologique». p 22. 
9. Sigmund Freud, «Le mot d'esprit...» opus cité, p 226.

10. Jacques Lacan, «L'instance de la lettre dans l'inconscient» in Les Écrits, Seuil, Paris 1966, p 508. conférence prononcée en 1957 à la Sorbonne.

11. Idem, p 515.

12. Lacan donne cette formule à la séance du 16 novembre 1969 sur «La logique du fantasme», séminaire inédit.

Jacques Lacan, «Radiophonie», in Silicet $n^{\circ} 2 / 3$, Seuils p. 58 .

13. Jacques Lacan, idem, p 63. 